\title{
Prácticas de cultura universitaria. El caso del ciclo de formación ciudadana de la Corporación Universitaria Minuto de Dios Sede Bello*
}

\author{
Elidio Alexander Londoño Uriza \\ Corporación Universitaria Minuto de Dios, Bello, Colombia \\ elidio.Iondono@uniminuto.edu.co \\ http://orcid.org/0000-0003-4625-3997 \\ Andrés Felipe Bedoya Bayer \\ Corporación Universitaria Minuto de Dios, Bello, Colombia \\ andresbayer@gmail.com
}

\section{RESUMEN}

El artículo analiza las apuestas que el Centro de Educación para el Desarrollo (CED) de la Corporación Universitaria Minuto de Dios (Uniminuto), sede Bello, realiza en materia del ciclo de formación ciudadana. El objetivo es valorar los esfuerzos que esa unidad académica adelanta para reforzar el desarrollo individual y social de sus estudiantes a partir de la apertura de espacios de reflexión ciudadanos, ético-políticos, cívicos, de convivencia y de consolidación de la democracia. El análisis se realiza a partir de los antecedentes que promovieron la creación del ciclo, las bases conceptuales que moderaron el tema de la ciudadanía en el CED, el inicio de las cátedras abiertas de ciudadanía,

las líneas temáticas que hicieron parte de esa estrategia, la conformación del ciclo de formación ciudadana, las alianzas estratégicas entre las apuestas de trabajo del CED para potenciar las actividades en materia de cultura ciudadana, y las perspectivas y orientaciones de trabajo del ciclo de ciudadanía. El estudio está respaldado en material primario recolectado del Archivo de Gestión del CED de la Uniminuto, sede Bello, y en bibliografía complementaria de carácter institucional y pedagógico en perspectiva de la educación para el desarrollo (EPD).

Palabras clave: cultura universitaria; prácticas educativas; formación ciudadana; Uniminuto; CED.

Cómo citar: Londoño Uriza, E. y Bedoya Bayer, A. (2021). Prácticas de cultura universitaria. El caso del ciclo de formación ciudadana de la Corporación Universitaria Minuto de Dios Sede Bello. Ciencias Sociales y Educación, 10(19), 55-84. https://doi.org/10.22395/csye.v10n19a3

Recibido: 7 de agosto de 2020.

Aprobado: 8 de octubre de 2020. 


\section{Practices of University Culture. The Case of the Citizenship Formation Cycle of the Corporación Universitaria Minuto de Dios, Bello Campus}

\section{ABSTRACT}

The article analyzes the Centro de Educación para el Desarrollo (CED or Center for Education towards Development, in English) of the Corporación Universitaria Minuto de Dios (Uniminuto) wagers about the citizen formation cycle. The paper assesses the efforts taken on by that academic unit to carry out the individual and social development of its students from the aperture of spaces for citizen, ethic-political, civic, coexistence and consolidation of democracy reflections. The analysis begins with the citizen formation cycle creation and background consolidation, the conceptual bases that moderated the citizenship topic in the CED, the citizenship conference principles, the thematic lines that took part of that strategy, the strategic alliances between the planned jobs of the CED to enhance the citizenship culture activities, and the cycle's perspectives and orientations. The study is supported by primary information consulted from the CED's archive management of Uniminuto's campus in Bello, and complementary bibliography of institutional and pedagogic character in the Education for Development (EfD) perspective.

Keywords: university culture; educational practices; citizen formation; Uniminuto; CED.

\section{Práticas culturais da universidade. 0 caso do ciclo de formação cidadã da Corpopação Universitária Minuto de Dios Sede Bello}

\section{RESUMO}

$\mathrm{O}$ artigo analisa as apostas que o Centro de Educação para o Desenvolvimento (CED) da Universidade Corporativa Minuto de Dios (Uniminuto) sede Bello faz em relação ao ciclo de formação do cidadão. O objetivo é avaliar os esforços que esta unidade acadêmica está realizando para reforçar o desenvolvimento individual e social dos seus alunos, começando pela aberturadeespaçosdecidadania,ética, política, reflexão cívica, convivência e consolidação da democracia. A análise parte do pano de fundo que promoveu a criação do ciclo, as bases conceituais que moderaram a questão da cidadania no DEC, o início das cadeiras abertas de cidadania, as linhas temáticas que faziam parte dessa estratégia, a formação do ciclo do treinamento do cidadão, as alianças estratégicas entre o trabalho do CED apostam na promoção de atividades no campo da cultura do cidadão e as perspectivas e orientações de trabalho do ciclo da cidadania. O estudo é apoiado por material primário coletado no Arquivo de Gerenciamento do CED da Uniminuto sede Bello e em bibliografia complementar de natureza institucional e pedagógica na perspectiva da Educação para o Desenvolvimento (EpD).

Palavras-chave: cultura universitária; práticas educacionais; formação cidadã; Uniminuto; CED. 


\section{Introducción}

La reflexión por los aportes que la Uniminuto, sede Bello (en cabeza del CED), adelanta en materia de formación ciudadana es bastante exigua dentro del campo de las humanidades y el sistema universitario colombiano. El trabajo que el CED de Bello lleva a cabo desde su conformación en 2006 ha significado la puntualización en los procesos de formación, investigación y proyección social de los estudiantes bajo el marco del fortalecimiento de principios como la responsabilidad social y el compromiso con la sociedad en su conjunto. Este esfuerzo ha valido para poner en interacción los fundamentos teóricos aprendidos en las aulas de clase con las prácticas cotidianas de la ciudadanía. Además, ha garantizado la promoción del desarrollo integral de la comunidad universitaria. Bajo ese escenario, el éxito de esas apuestas ha estado representado en los aprendizajes individuales y colectivos de los actores que han hecho parte de los procesos. Sin embargo, los resultados apenas son visibles para la academia, ya que la poca divulgación de las estrategias y procedimientos adelantados no han permitido visibilizar las contribuciones que se han ido desarrollando alrededor de la educación cívica y el ejercicio de la ciudadanía.

La gestión del CED de Bello ha posibilitado diseñar estrategias académicas orientadas al liderazgo social y comunitario del Valle de Aburrá y el Departamento de Antioquia. Uno de los objetivos principales de este centro ha sido facilitar la formación de las habilidades, competencias y capacidades de los futuros profesionales en el ámbito de los derechos humanos, valores, participación, concepción de lo público-privado, tolerancia, paz, entre otras dimensiones de lo civil y lo cívico. Esas apuestas contribuyen al fortalecimiento de las relaciones interinstitucionales con entidades no gubernamentales como fundaciones, corporaciones y asociaciones; además de instituciones de educación básica, secundaria y media a nivel local y regional: aliados que han participado del intercambio de experiencias suscitadas dentro de los procesos de formación en ciudadanía. En ese sentido, las acciones adelantadas por el CED se han ido convirtiendo en espacios que incentivan la promoción del desarrollo integral de las personas que han hecho parte de sus áreas de trabajo, o se han vinculado en los procedimientos que buscan aportar a la transformación de las comunidades y a la solución de los problemas sociales. Todos esos aspectos son una clara muestra de la importancia de las estrategias de trabajo del CED de Bello; no solo por el respaldo institucional que ha adquirido con las organizaciones sociales, sino también porque su experiencia soporta, motiva y proyecta la formación de los estudiantes en el ámbito de la ciudadanía.

Es de anotar, teniendo en cuenta el Acuerdo 02 (2014), que los lineamientos generales para la proyección social del sistema Uniminuto (conformado por siete estrategias: i) práctica profesional, ii) práctica en responsabilidad social, 
iii). voluntariado, iv) educación continua, v) relacionamiento con graduados, vi) articulación de la educación media y vii) transferencia de conocimientos a través de proyectos) responsabilizan al CED de los ítems ii y iii (Vicerrectoría General Académica, 2018, p. 25). No obstante, este centro también está llamado a fortalecer la estrategia de educación continua (que direcciona los talleres, charlas cursos, seminarios, cátedras, entre otros) para asumir

\begin{abstract}
la labor de educar al ser humano durante toda la vida y responder de manera continua a los desafíos de las nuevas problemáticas y las nuevas formas de conocimiento en un mundo cambiante, tal como lo promueve la Unesco. Comprende las alternativas y opciones de formación sin pre-requisitos [sic] ni tendientes a titulación. La educación continua permite fortalecer las competencias en temas específicos de interés en diversos públicos, con metodologías presenciales o virtuales. (Corporación Universitaria Minuto de Dios, s. f.)
\end{abstract}

Si bien es cierto que el CED no es una facultad que ofrece programas académicos de pregrado y posgrado para el aprendizaje de estudiantes, sus áreas de formación y líneas de acción son trabajadas en el marco de la responsabilidad social y la formación ciudadana. Cada línea de acción es un aporte al fomento del desarrollo humano en sus dimensiones personales, cívicas y profesionales. Precisamente, una de las acciones que permiten potenciar las líneas de acción del CED es el ciclo de formación ciudadana: un espacio que promueve el diálogo intercultural, el establecimiento de valores cívicos y la conformación de una conducta ciudadana democrática fundamentada en la participación y cooperación para reconocer la realidad, el pensamiento crítico y reflexivo, así como las acciones ciudadanas orientadas al cambio social. Estos aspectos trascienden la concepción minimalista - representada por el ejercicio del voto y la obediencia a las leyes - y pone en escenario la promoción de la formación ciudadana desde una concepción maximalista - fundamentada en la participación activa desde un compromiso y orientación pública integral en todas las esferas de la sociedad—, según la distinción que hizo Terence H. McLaughlin (1992) entre la educación cívica y la educación ciudadana. En realidad, esos enfoques también van en consonancia con los planteamientos que Adela Cortina-Orts (2010) realizó cuando separó la ciudadanía simple —expresada en una igualdad que elimina las diferencias y el pluralismo social— de la ciudadanía compleja — caracterizada por incluir las diferencias étnicas y religiosas, y las tendencias sexuales, ambientalistas, ecologistas, artísticas, políticas, ideológicas, entre otros-, para promover la expresión de todas las voces que integran a la sociedad.

Por eso, el análisis de caso que proponemos supone valorar y repensar los parámetros de docencia, trabajo institucional y modelo de formación ciudadana que el CED de Bello está llevando a cabo desde su creación. Este ejercicio también sirve para ampliar la observación crítica sobre los diferentes esfuerzos que 
se están adelantando en el país en materia de educación cívica y reconocer las fortalezas y vacíos que ese tipo de apuestas puede presentar en el ámbito de la educación superior. Es, además, la oportunidad para explorar los aportes que el sistema Uniminuto realiza para promover el compromiso y la corresponsabilidad (desde el fomento de las actitudes y valores en el marco de la ciudadanía) en una de sus sedes ${ }^{1}$.

La apuesta de reforzar la educación cívica en la sede de Bello de la Uniminuto, a partir de la implementación del ciclo temático, ha presentado tal aceptación institucional que ya son más habituales las cátedras abiertas de ciudadanía, la existencia de proyectos de extensión y voluntariado, así como la realización de diferentes procesos de proyección social que buscan contribuir a la comprensión y solución de los principales problemas de los agentes y sectores sociales (e institucionales) que hacen parte de la dinámica de interacción e integración de la institución universitaria.

Iniciemos entonces con los antecedentes que dieron origen a la implementación del ciclo de formación ciudadana en Uniminuto, sede Bello, el contexto que posibilitó su creación, las razones que motivaron su conformación y los componentes que perfilaron su estructura formativa para abordar las bases conceptuales que soportaron su consolidación, ya que con su diseño se refuerza el trabajo de educación cívica en la sede.

\section{Antecedentes y contexto}

La importancia de la formación ciudadana en el CED, y en Uniminuto en general, se sustenta en el impulso de una ciudadanía global caracterizada por conocimientos, actitudes y valores propios de una cultura solidaria que garantice el compromiso por lo social, el desarrollo humano, la apertura del crecimiento económico sostenible y la lucha contra la pobreza y la exclusión. Todo ello se postula bajo dimensiones cívicas y civiles que promuevan la formación de ciudadanos capaces de imaginar y proponer nuevos órdenes de agenciamiento, hegemonía y liderazgo. Para esos efectos, el aprendizaje sobre lo cívico — constituye la participación política formal mediante mecanismos e instituciones de democracia representativa establecidos a través de lazos débiles entre otros distantes, geográfica o ideológicamente, que permiten la coexistencia democrática desde los bienes comunes y más allá de las diferencias-y lo civil —expresado en la convivencia y los derechos de libertad personal, de expresión, de propiedad y de celebrar contratos en el dominio privado- es fundamental para lograr la

En la actualidad, Uniminuto está conformada por las rectorías y vicerectorías: Antioquia y Chocó, Atlántico, Bolívar, Bogotá presencial —calle 80- virtual y a distancia, Bogotá Sur, Cundinamarca, Eje Cafetero, Huila, Llanos Orientales, Nariño, Norte de Santander, Santander, Tolima, Valle y Magdalena. 
consolidación de sujetos ciudadanos capaces de abordar los cambios sociopolíticos y culturales que demandan las nuevas realidades (Riquelme-Muñoz, 2018).

En ese sentido, el diseño de propuestas que permitan abrir espacios de formación y prácticas culturales cívicas en series temáticas relacionadas entre sí es una apuesta que ha potenciado los esfuerzos del CED por consolidar la educación ciudadana. Las bondades de ese tipo de estrategias están representadas en el contacto que estudiantes, docentes y administrativos tienen con profesionales en ejercicio, quienes contribuyen al proceso de proyección de los jóvenes bajo las experiencias académicas, profesionales, laborales y vivenciales que comparten en esos espacios. Ese tipo de prácticas promueve la participación de la población universitaria, estimulan la reflexión crítica sobre los diferentes problemas de la sociedad, fomentan conciencia ciudadana, impulsan el potencial humano y generan conocimiento diverso que influyen en el desarrollo de las competencias personales, familiares y laborales de los futuros profesionales del país.

Es importante advertir que el CED de Bello no solo imparte los cursos de Desarrollo Social Contemporáneo y las Práctica de Responsabilidad Social, como ocurre en los demás CED de Uniminuto. Los cursos que hacen parte de la unidad están insertos en tres momentos de aprendizaje transversal: pensamiento social, desarrollo local y formación para la ciudadanía. La primera se encarga del componente de formación humana y está conformada por las asignaturas: Cátedra Minutos de Dios, Liderazgo en Valores, Proyecto de Vida, Humanidades, Actividad Física y Desarrollo Humano como Forma de Felicidad; la segunda centra su atención en la responsabilidad social y comprende los cursos: Responsabilidad social. Una práctica de vida y Práctica en responsabilidad social y desarrollo social contemporáneo; y la tercera aborda la línea de formación ciudadana desde las materias Constitución Política, Resolución de Conflictos, Ética Profesional, Teoría del Conflicto, Memoria y Paz, Cátedra de la Paz y Electivas CMD (que aporta a la formación integral y está sujeta al componente Minuto de Dios). La labor de docencia sobre esos tres momentos se complementa con las funciones sustantivas de investigación, proyección y extensión que desarrollo el CED. Las actividades que hacen parte de las funciones de proyección están representadas en el agenciamiento, coordinación y acompañamiento al desarrollo de las Prácticas de responsabilidad social (PRS); además del liderazgo en el voluntariado y el fortalecimiento de la educación continua. Para el caso del programa de voluntariado, las tareas se concentran en promover el compromiso individual y colectivo de los estudiantes hacia las demás personas (con acciones generosas) para fomentar un desarrollo culturalmente plural, socialmente justo y ecológicamente sostenible. Mientras tanto, el ciclo de formación ciudadana (que hace parte de las acciones más relevantes de la educación continua) busca potenciar en los estudiantes (y la comunidad universitaria en general) las 
dimensiones sociocríticas a través de cátedras, debates, cineforos, talleres experienciales y eventos de liderazgo en valores.

El ciclo de formación ciudadana tiene su origen epistemológico en las bases teóricas del CED, el cual se ha fundamentado en las transformaciones que la educación para el desarrollo (EPD) produjo para el mejoramiento de la educación. Recordemos que la EPD es una propuesta pedagógica que tiene fuerte influencia de la educación popular. Según Paula Botero Carrillo, Ana Yudy Morán Matiz y Rigoberto Solano Salinas (2010), esa influencia transformó los roles de participación de los docentes en el proceso formativo, ya que sus fronteras dejaron de estar limitadas a las aulas de clase. En este sentido, la investigación y la incidencia política empezaron a ser parte de sus espacios de actuación universitaria. El cambio en las funciones sustantivas de los educadores animó los esfuerzos por establecer una pedagogía crítica que no estuviera mediada por los principios jerárquicos profesados en la educación tradicional (que ubicaba al docente en la punta de la pirámide y posicionaba a los estudiantes en la base). A partir de este tipo de modificaciones, las clases magistrales pasaron a un segundo plano, mientras que las actividades en el aula comenzaron a generar alternativas de aprendizaje que pusieron en interacción horizontal la relación educador y educando. Se dio lugar, en este contexto, a ejercicios pedagógicos como "talleres, salidas de campo, análisis de contextos o problemas cercanos", entre otros (Botero Carillo, Morán Matíz y Solano Salinas, 2010, p. 17; Freire, 1972). Bajo ese marco, el objetivo fue claro: construir procesos de reflexión que garantizaran el pensamiento autónomo y consciente de la población estudiantil, y la comunidad universitaria en general, a partir del reconocimiento de las opciones éticas y políticas propias que contribuyen al establecimiento de un desarrollo social transformador de las realidades y los contextos.

Con la implementación de la propuesta pedagógica de la EPD, el horizonte de acción del trabajo formativo se amplió a otros escenarios que pusieron en diálogo a la población universitaria con la sociedad. Así, la participación de los estudiantes y docentes dejó de ser exclusiva dentro del proceso pedagógico. Líderes de comunidades y organizaciones sociales, integrantes de colectivos vulnerables, miembros de organizaciones de mujeres, grupos étnicos, asociaciones ambientalistas, personas en condición de discapacidad, víctimas, entre otros, empezaron a ser considerados como sujetos de aprendizaje por el carácter colectivo y dialéctico del proceso formativo. El diálogo entre el conocimiento adquirido en las aulas de clase - que incluye el conocimiento letrado y la enseñanza experiencial - y los saberes populares fueron propulsores del aprendizaje cívico, crítico, activo y socialmente responsable de las personas involucradas dentro del proceso de formación, el cual termina insertándose en los movimientos sociales, las características emocionales propias 
de la humanidad y en los ámbitos de la cotidianidad. Cabe agregar que el modelo de la EPD ha tenido variaciones históricas que han repercutido en el modo de entender el desarrollo en el CED de la Uniminuto, a saber, el enfoque caritativo-asistencialista dado entre 1945 y 1950, la perspectiva desarrollista y de educación para el desarrollo evidenciado entre 1960 y 1970, el enfoque de una educación para el desarrollo crítico y solidario trabajado entre 1970 y 1980, la línea de la educación para el desarrollo humano y sostenible realizada entre 1980 y 1990 - configurada en un período de cambio caracterizado por la crisis del desarrollo, el esfuerzo dialéctico entre la paz y los conflictos, la incentivación de la dimensión del género, el reconocimiento del medio ambiente y los límites del desarrollo, los esfuerzos sobre el comercio justo y consumo consciente, la búsqueda de la superación del racismo, el incremento de las migraciones poblacionales y el énfasis en la confluencia de las educaciones para el desarrollo y la paz- y el enfoque humanista que facilitó el abordaje de la educación para la ciudadanía global desde 1990 hasta la actualidad (Mesa Peinado, 2000, p. 8; Solano Salinas, 2011, p. 95).

Es de anotar que el CED del sistema Uniminuto está conformado por unidades académicas dependientes a las sedes (o seccionales) de la institución. No obstante, la razón de ser de cada una es homogénea y responde a tres momentos de aprendizaje organizados en pensamiento social, formación en responsabilidad social y desarrollo comunitario. Estos momentos están estructurados en las funciones sustantivas de la institución: la docencia, la investigación y la proyección social. Sin embargo, las estrategias de trabajo de cada CED son independientes y obedecen a las coordinaciones y direcciones de cada centro. En ese sentido, el fundamento conceptual y epistemológico que el CED de Uniminuto recogió de la EPD y la educación popular es transversal y aplica para todas las unidades en cada una de las sedes. Desde esas bases epistémicas, la vinculación entre la academia y las comunidades — que hace parte de las proyecciones del sistema universitario- empezaron a modelar los frentes de trabajo de las unidades, lo que facilitó el esfuerzo por la comprensión de las realidades sociales, a partir de la labor constructiva de conocimiento con relevancia social, y estimuló el proceso de aprendizaje desde la formación humana y el compromiso por la trasformación social y el desarrollo sostenible.

Ahora bien, las variaciones del modelo de la EPD, representadas en generaciones, influenciaron el trabajo de la sede de Bello de la Uniminuto desde cinco hitos históricos: i) la creación del Centro de Práctica Social entre 1998 y 2001, el cual estuvo encargado de la formación del estudiantado en lo tocante a la responsabilidad social; ii) la conformación del CED y la reflexión académica sobre el discurso del desarrollo social en asignaturas como Fundamentos teóricos de la práctica social entre 2006 y 2008; iii) la implementación del curso Desarrollo Social 
Contemporáneo desde el enfoque de la EPD y el modelo praxeológico en perspectiva del aprendizaje ciudadano socialmente responsable; iv) la creación de la ruta de formación ciudadana entre 2009 y 2011 (que retomaba el enfoque humano y social de la EPD y los aportes de Solano Salinas en esa materia); y v) la transición de los componentes humano y social a la incorporación del biodesarrollo, las problemáticas ambientales, la perspectiva de género, la interculturalidad, la no violencia y el "buen vivir" desde la publicación del libro De-construyendo la educación para el desarrollo. Una mirada desde Latinoamérica en el año 2012 (Orrego Echeverría, 2014, p. 167). Como sugiere Orrego Echeverría (2014), y teniendo en cuenta esa publicación, la profundización en las bases teóricas y temáticas de la EPD y la noción de desarrollo supuso la revaloración de los sentidos, apuestas, teleologías y epistemologías de la comprensión intercultural-crítica de la EPD y el desarrollo. A partir de esos elementos, el perfil de la formación ciudadana, en congruencia con la EPD y la dialéctica de la educación popular, en el CED de Bello empezó a definirse desde el esfuerzo por incentivar una ciudadanía activa que se antepusiera a las ciudadanías restringidas propias de los regímenes democráticos con restricciones a los derechos sociales.

En otras palabras, la conformación del Centro de Práctica Social y el CED de Uniminuto, sede Bello, fueron el punto de partida para que la responsabilidad social y la formación ciudadana tuvieran mayor presencia en la sede. El Acuerdo 224 de (2006), que cambió el Centro de Práctica Social por el CED, abrió el espacio para que desde esa unidad académica se realizaran acciones que garantizaran la práctica social, la formación en responsabilidad social y la promoción del "desarrollo integral de las personas, organizaciones y comunidades" (Corporación Universitaria Minuto de Dios, 2006, p. 1). Con esa unidad, los procesos pedagógicos de sensibilización y aprendizaje incorporaron estrategias de promoción ciudadana relacionadas con las actitudes y valores propios del humanismo cristiano, la espiritualidad eudista ${ }^{2}$ y, en general, la filosofía de la Obra Minuto de Dios liderada por el sacerdote Rafael García Herreros Unda desde 1946 para trabajar por una "nación justa, equitativa y en paz" (Organización El Minuto de Dios, 2013, p. 1). Esas directrices misionales buscaron efectuarse en clave de la EPD. Con ese enfoque pedagógico, el proyecto de formación ciudadana y democrática empezó a implementarse desde el impulso a la construcción de procesos de reflexión que estimularan el pensamiento global, la actuación local, la participación en el desarrollo de las comunidades —y el mundo en general-y la lucha contra la pobreza y la exclusión. En ese sentido, el perfil del ciudadano formado por el CED debía incluir criterios suficientes para construir una sociedad

Recordemos que la espiritualidad eudista surge en el siglo XVII con San Juan Eudes bajo la Congregación de Jesús y María, y está caracterizada por: i) la dimensión humana, ii) la dimensión espiritual, iii) la dimensión comunitaria y iv) la dimensión intelectual. Todos ellos se fundamentan en el cristocentrismo, la experiencia de vida y reino y la evangelización-formadora. 
civil que permitiera su opinión e incidencia en los temas económicos, políticos y sociales; además de garantizar el amparo de los derechos humanos, la conformación de una ciudadanía mundial y la cimentación de una sociedad equitativa (Corporación Univeristaria Minuto de Dios, 2006).

Desde su fundación, el cuerpo administrativo del CED no ha variado sustancialmente. El primer director que lideró los destinos de la unidad fue el padre Orlando de Jesús Hernández Cardona en el período 2007-2011, el segundo fue Carlos Andrés Monsalve Roldán entre 2015 y 2016 (quien también había sido coordinador general desde 2006) y el tercero fue Andrés Felipe Bedoya Bayer entre 2017 y 2019. En el período 2016-2017, el cargo directivo quedó vacante y las actividades ejecutivas fueron lideradas por el vicedirector de la sede ${ }^{3}$, Jorge Arbey Toro Ocampo, y la secretaria académica Catalina María Sepúlveda Zapata. Para el año 2020, Toro Ocampo volvió a asumir funciones como director encargado, pero bajo el acompañamiento de la secretaria Ilba Patricia Rodríguez Escobar. Todos ellos han aportado al fortalecimiento del trabajo que la sede ha llevado a cabo en materia de responsabilidad social en personas y organizaciones, han contribuido al desarrollo comunitario desde la gestión de proyectos de impacto social y han generado reflexiones y apuestas sobre las realidades sociales y económicas en el ámbito local, nacional e internacional. Asimismo, y a pesar de las diferencias de cada administración, el análisis a los modelos predominantes y alternativos ha estado vigente, pues siempre se ha propendido por la búsqueda de competencias ciudadanas que faciliten la generación de dinámicas pedagógicas innovadoras y eficientes de acuerdo con los principios de la Uniminuto, esto es, promover la valoración de las diferencias, reconocer la igualdad de los derechos entre las personas y fomentar la comprensión de la importancia de cumplir las leyes. En suma, estos esfuerzos son concebidos dentro de la línea de la EPD bajo enfoques pedagógicos, críticos y emancipadores que se apoyan en la lectura contextual y la concientización de los sujetos y colectivos para incentivar la promoción de agentes de transformación de entornos socioculturales, políticos y económicos.

\section{Bases conceptuales y epistémicas de la ciudadanía en el CED}

Luego de la conformación del CED en 2006, el modelo de formación ciudadana que empezó a utilizarse fue el que diseñó el Ministerio de Educación Nacional entre 2002 y 2005 para el sistema de educación básico y medio. Ese modelo estuvo basado en la búsqueda de competencias ciudadanas que garantizaran el fomento de los conocimientos cognitivos, emocionales y comunicativos necesarios para alcanzar una sociedad democrática. Así, los estudiantes debían saber y saber hacer para ejercitar las habilidades adquiridas en cada uno de

Es de resaltar que la sede Bello hace parte de la rectoría Antioquia-Chocó. 
sus hogares, medios escolares y contextos que hacían parte de su cotidianidad. En ese orden de ideas, el desafío de formar para la ciudadanía promovió la convivencia pacífica, la participación responsable, el concurso constructivo en los procesos democráticos, así como el respeto y la valoración de la pluralidad y las diferencias en los entornos cercanos, comunitarios, nacionales e internacionales (Chaux, 2004, p. 10). Aunque el público objetivo de ese modelo era el niño o niña de cada institución de educación secundaria y media, sus bases conceptuales y metodológicas sirvieron para ser replicadas en el CED a partir de herramientas que incentivaran el relacionamiento con los otros desde una perspectiva comprensiva y justa que posibilitara la resolución de problemas cotidianos. Un formato que sirvió de referencia para las actividades que fueron adelantadas por el CED entre 2006 y 2009 en lo tocante a la formación ciudadana. Empero, y teniendo en cuenta la ausencia de un modelo formativo que respondiera al enfoque de educación para el desarrollo, Morán Matiz (2010, p. 108) llevó a cabo la investigación Un modelo integral de formación ciudadana para diseñar un esquema conceptual y pedagógico que direccionara los procesos de manera práctica y aplicable a la construcción de una ciudadanía crítica y activa que respondiera a las exigencias ciudadanas que demanda el mundo contemporáneo.

El producto que consolidó esa investigación fue la Caja de herramientas para la formación ciudadana (Morán Matíz, Donato Hernández e Izquierdo Ramírez, 2009 , p. 124). Esta publicación consta de cuatro módulos para trazar la ruta metodológica más adecuada en las fases del modelo, cuyas fases están representadas en: i) lo que debemos saber para ser ciudadanos críticos y activos, ii) cómo ser ciudadanos reflexivos y críticos, iii) nos autoconocemos y ponemos en el lugar de los otros para ser ciudadanos activos y iv) somos ciudadanos asertivos y socialmente responsables. Ese material prioriza en temáticas como

la pobreza, la desigualdad, la exclusión, la injusticia social, las diferentes expresiones de violencia, la discriminación, la intolerancia [...], la noviolencia [sic], el respeto a la diferencia, la equidad de género, la acción colectiva, la cohesión social, la ecología y la incidencia política. (Morán Matíz, Donato Hernández e Izquierdo Ramírez, 2009, pp. 3-4).

Cada tema reverbera criterios básicos de responsabilidad social universitaria y estimula la formación de sujetos políticos a través de la educación para el desarrollo. Desde esa producción bibliográfica, las estrategias de trabajo del CED se fueron perfilando y afianzando hacia el reconocimiento de la dignidad humana y la potenciación del tejido intercultural de la sociedad, enlazados entre lo local y global. De hecho, Moya Garzón (2012) también publicó el libro ¿Cómo va la formación ciudadana? Seguimiento a la implementación del modelo de formación ciudadana del CED en Uniminuto para repensar la pertinencia teórica, conceptual, pedagógica y metodológica del modelo proyectado por la Caja 
de herramientas para la formación ciudadana y la efectividad de las apuestas reflexivas (y de construcción colectiva) que suscitó su agenciamiento en los claustros universitarios de la Uniminuto. Con esta última publicación, Morán Matíz (2011) pretende revalorar el modelo y abrir camino a la actualización constante de los procesos. En este sentido, considera las etapas que la conforman: conocer, cuestionar, emocionarse, incidir en la realidad y buscar establecer la relectura de la realidad, la toma de posiciones y la generación de propuestas de transformación social.

Sin duda, los aportes teóricos, metodológicos, conceptuales y epistémicos de Mesa Peinado (2000), Botero Cariilo, Morán Matíz y Solano Salinas (2010), Orrego Echeverría (2014), entre otros, han convertido su trabajo en los principales referentes de la formación ciudadana en los CED de Uniminuto. Las apuestas, estrategias y actividades que desarrolla cada unidad fundamentan sus acciones desde los elementos de reflexión que aportan para potenciar las experiencias y los alcances en los procesos de educación, aprendizaje y acción. Aunque la gran mayoría de los autores hayan hecho su trabajo enfocado hacia la práctica en responsabilidad social (en particular Morán Matiz), los presupuestos que plantean también contribuyen a la base conceptual de la formación ciudadana, pues permiten considerar las necesidades, intereses y contextos de los actores que hacen parte de los procesos. Ello hace que la reflexión y la construcción de las ciudadanías se promueva de manera global bajo alternativas que faciliten el entendimiento de los entornos sociales, las repercusiones de las problemáticas actuales en materia de desigualdad e injusticia social, así como la relación local-global que influye en las dinámicas de gobierno, Estado y cultura. Estos temas son de primer orden porque convocan la deconstrucción de la sociedad y la priorización en aforos relacionados con la inclusión social, el desarrollo de la civilidad, la convivencia, la consolidación de la democracia y la construcción de lo público en el orden del desarrollo local.

\section{Creación de las cátedras abiertas de ciudadanía}

Las apuestas que el CED de Bello realizó entre 2008 y 2010 por la apertura de espacios socioeducativos para fomentar la comprensión crítica de sus territorios, así como la construcción conjunta de acciones cotidianas de transformación social, estuvieron mediadas por las PRS. Según José Ayner Valencia Rivas (2013, pp. 84-85), para ese período las propuestas de acción de la unidad carecían de articulación entre la institución universitaria, el estudiantado y los contextos de las instituciones vinculadas; el marco conceptual y metodológico todavía era débil; la propedéutica entre los cursos ofrecidos y la reflexión académica era bastante incipiente; y la implementación de las estrategias praxeológicas requerían de un notable mejoramiento. Esas debilidades eran esperables, pues los escasos años 
de funcionamiento del CED auspiciaban aspectos por mejorar en los procesos. Lo cierto del caso es que las competencias en responsabilidad social y formación ciudadana estaban relacionadas con las PRS. El objetivo era complementar el aprendizaje ciudadano y de corresponsabilidad que los estudiantes habían adquirido en las instituciones educativas que los había egresado. En este sentido, se buscó reforzar la conciencia en ciudadanía y el ejercicio cívico como piezas garantes del desarrollo de las capacidades críticas y reflexivas bajo prácticas cotidianas que trascendieran el escenario universitario.

Las apuestas por fomentar el aprendizaje ciudadano en el CED de Bello empezaron a ser más preponderantes durante los años 2011 y 2012. La publicación de la Caja de herramientas para la formación ciudadana (Morán Matíz, Donato Hernández e Izquierdo Ramírez, 2009) y el libro ¿Cómo va la formación ciudadana? (Moya Garzón, 2012) dieron elementos importantes para cimentar las actividades formativas en temas de ciudadanía. Además, la implementación del módulo de competencias ciudadanas en las pruebas Saber Pro de educación superior (liderada por el Ministerio de Educación Nacional y efectuada en el examen de junio 3 de 2012) ${ }^{4}$ reafirmó la importancia de la formación ciudadana en los claustros universitarios. Por eso, el CED de Bello intensificó sus estrategias de trabajo en esa área haciendo énfasis en la formación integral y la búsqueda de la excelencia humana, profesional, competente, ética y comprometida con la sociedad y el desarrollo sostenible. Estos temas incentivaron el fuero de sensibilización, problematización, concienciación y acción adelantadas por la unidad académica, en consonancia con la misión propia de la institución (Solano Salinas, 2011). Para ese momento, y como sugiere Yamile Rodas Restrepo, las actividades no tenían un carácter de institucionalidad, no había una técnica de planeación consolidada que las liderara y el perfil metodológico se hacía desde el aprendizaje experiencial (comunicación personal, 12 de abril de 2019). No obstante, esas primeras apuestas lograron ubicar a los estudiantes en sus realidades, sirvieron para centrarlos en sus necesidades desde una posición con miras a la transformación, facilitaron su reconocimiento mutuo, permitieron que su responsabilidad como sujetos de derechos y actores políticos fuera abordada con mayor conciencia, abrieron campo al pluralismo y la deliberación como mecanismos de acuerdos que promueven el bien común y proporcionaron el reconocimiento de la otredad, el pensamiento del otro y la dilucidación del ser humano.

Precisamente, y como evidencia de los alcances de esas actividades, para el primer semestre de 2013 la formación ciudadana en el CED empezó a ser expre-

4 Recordemos que estas pruebas son un requisito de grado en Colombia para los estudiantes que adelantan programas de pregrado en instituciones de educación superior y han cursado, como mínimo, el $75 \%$ de sus créditos académicos. En ellos se evalúan las siguientes competencias genéricas: lectura crítica, razonamiento cuantitativo, competencias ciudadanas, comunicación escrita e inglés y temáticas con contenidos específicos relacionados al área de formación profesional de cada estudiante. 
sada en cátedras abiertas de ciudadanía. Los docentes Edgar Darío Hernández Parra y Nidia Janeth Ramos Agudelo lideraron esa iniciativa y propusieron implementar unos conversatorios lúdicos que originaran espacios pedagógicos direccionales de sana convivencia. Así, los estudiantes de primero, segundo y tercer semestre fueron convocados para participar en esos escenarios. El primero fue un "bosque mágico" que contenía un picnic con un reglamento de participación universitaria, un oráculo de ángeles que denotaba el reglamento interno de trabajo de los estudiantes, educadores, administrativos y directivos, y la posibilidad de conformar colectivos que emularan los grupos que hacen parte de la sociedad: equipos deportivos, culturales, musicales, de negritudes, de diversidades (en particular los LGTBI) y de teatro (representado en el grupo Misión 21). El segundo espacio (o cátedra abierta) fue llamado UniCity para figurar las variables interpretativas de la humanidad y la ciudadanía que permitieran reconocer la importancia de la defensa de los derechos de las comunidades, la búsqueda de una sana convivencia y el respeto de las diversidades sexuales y de género. Todos ellos se expresan en colectivos (conformados por jóvenes y adultos entre dieciséis y treinta y ocho años) que buscaron formular estrategias garantes de movilización y visibilización de los estudiantes hacia la institución universitaria. Esos ejercicios, junto con la aplicación experiencial de los conocimientos adquiridos y la construcción de sociedad a partir de los procesos de participación activa, proporcionaron la formulación de mecanismos de empoderamiento basados en un pensamiento crítico-constructivo de desarrollo integral, personal y social (Ramos Pino, Ortiz Palacio y Gómez Cardona, 2013, pp. 6-7).

Las jornadas de esas actividades se llevaban a cabo en semanas programadas que dieron cabida a la conformación de espacios de debate y construcción colectiva, lo que permitió recoger la voz de los estudiantes frente a sus análisis sobre las problemáticas de la sociedad colombiana. El objetivo de ese tipo de espacios era fortalecer las competencias requeridas para la presentación de las pruebas Saber Pro. Pero, en realidad, la participación en esos escenarios buscaba que los estudiantes reforzaran temas de ciudadanía como un aporte a su proceso formativo. Desde ese horizonte, las temáticas que más fueron objeto de discusión versaban alrededor de la sana convivencia, la constitución política, la multiculturalidad, los derechos y deberes de las comunidades y grupos colectivos (como los LGTBI, afrodescendientes, víctimas del conflicto armado colombiano, entre otros), los mecanismos de participación ciudadana y la aplicación de las pruebas de competencia. De tal suerte, las cátedras empezaron a configurar un proscenio que apuntaba a la conformación y consolidación de valores ciudadanos desde el reconocimiento de las condiciones del contexto histórico y político colombiano. 
La vinculación de las cátedras a las estrategias de trabajo del CED facilitó que las apuestas de aprendizaje en ciudadanía fueran mucho más integrales en los procesos. Además, el formato de las cátedras era un espacio de comunicación y valoración de saberes en el que los estudiantes demostraban su avance académico en materia de formación ciudadana. Por ello, durante los primeros dos años (2013-2014), los ponentes eran los estudiantes que estaban cursando las asignaturas del CED (en particular el curso de Desarrollo Social Contemporáneo y el de Práctica en Responsabilidad Social) y hacían parte de los programas de psicología, trabajo social o comunicación social y periodismo (Y. Rodas, comunicación personal, 12 de abril de 2019). En efecto, el esquema de las cátedras fue tomando forma para animar la discusión académica sobre temáticas como el conflicto armado colombiano, los derechos de las comunidades negras e indígenas, la configuración de la democracia mediante procesos de reinserción, la ética ambiental, los acuerdos humanitarios, la búsqueda de la paz, la participación universitaria y ciudadana, los mecanismos de participación, el género y las subjetividades, la convivencia cívica, entre otros. Todas estas líneas de análisis estimulan el cambio de la realidad local y global para conformar un mundo más justo, equitativo, respetuoso, diverso y con consideración con el medio ambiente.

Con todo lo mencionado, los avances del CED en materia de formación ciudadana eran innegables. De hecho, en el segundo semestre de 2015 fue llevado a cabo el Diplomado en Ciudadanía y Liderazgo Social. Este espacio académico buscó actualizar y fortalecer los conocimientos de los estudiantes alrededor de la ciudadanía, la relación conceptual entre el sujeto crítico-reflexivo, la importancia de la generación de preguntas sobre la realidad propia y la continuidad que debe haber entre los elementos teóricos y la observación práctica. Con ello, se buscó que los participantes tuvieran fundamentos sobre las acciones transformadoras, el horizonte del liderazgo desde una apuesta ético-política conectada con la responsabilidad ciudadana, las diversas maneras de elegir a los líderes con sus respectivas consecuencias dentro del trabajo colectivo y el fortalecimiento de los argumentos a través de procesos comunes de organización del trabajo (Estudiantes del Diplomado, 2015). Ese escenario de aprendizaje complementó la labor realizada desde las cátedras de ciudadanía y dio luces para perfilar los esfuerzos del componente de formación ciudadana.

Para el año 2014, la estructura de planeación anual del CED de Bello fue replanteada. Así, las estrategias de su quehacer fueron orientadas a la conformación de cinco macroproyectos que abarcaron las tres áreas de trabajo de la unidad: pensamiento social, desarrollo local y formación para la ciudadanía. Esa revaloración de la práctica propendió por la consolidación de la articulación entre la docencia, la investigación y la proyección social. Los cinco macroproyectos fueron denominados en su conjunto "sombrillas" (por las características que esa 
expresión tenía para contener la diversidad tipológica de los esfuerzos realizados por el CED). Los nombres que recibió cada uno correspondieron a: "Proyecto 1: consolidación del CED", "Proyecto 2: fortalecimiento del voluntariado", "Proyecto 3: escuela de paz y bio-desarrollo", "Proyecto 4: competencias ciudadanas" y "Proyecto 5: fortalecimiento de organizaciones sociales" (Corporación Universitaria Minuto de Dios, 2015, p. 86). Para el caso de las competencias ciudadanas del proyecto 4 , las actividades fueron divididas en:

a. Actualización de las cartas descriptivas de los cursos de CMD a partir de la incorporación de elementos conceptuales a la luz de la constitución política.

b: Implementación de tres cátedras de ciudadanía.

c. Promoción, diseño y ejecución de las Olimpiadas de Ciudadanía.

d. Actualización docente en temáticas relacionadas con la resolución de conflictos y constitución política.

e. Taller de profundización en constitución política de Colombia para estudiantes de la seccional Bello.

f. Seguimiento y evaluación del proyecto.

Sin embargo, de esas seis actividades solo los puntos B, C y F pudieron llevarse a cabo de manera efectiva. De hecho, con esa estructura se fue consolidando el apodado "proyecto de ciudadanía" (como también se conocía a la estrategia de competencias dentro del argot del equipo de colaboradores del CED para ese momento). Lo cierto es que el macroproyecto de competencias ciudadanas dispuso las condiciones para que las cátedras aportaran a la formación ciudadana de los estudiantes y complementara la construcción de civilidad con temas de orden social, cultural, político, ambiental y de memoria que facilitaran la reflexión sobre la dignidad, la libertad, la justicia, la paz y la vida (Centro de Educación para el Desarrollo, 2014). En efecto, y con la apuesta de gestión expresada en macroproyectos, la aplicación de las cátedras respondió a la intencionalidad de complementar la formación reflexiva, crítica y propositiva de la comunidad estudiantil. Es este sentido, las cátedras aportaron a los esfuerzos del CED por orientar los procesos formativos de la Uniminuto, sede Bello, hacia el desarrollo humano integral y la generación de una cultura política allanada al reconocimiento de la interculturalidad y al ejercicio franco de la ciudadanía. Esos esfuerzos también eran complementados con cursos específicos como el Diplomado en Ciudadanía y Liderazgo Social que ofreció el CED de Bello para ese semestre. Así, fue un espacio de actualización de los conocimientos y competencias propias de los participantes (que estimulaba la interacción con sus comunidades) desde la potenciación de sus liderazgos y la promoción de procesos de 
participación ciudadana activa adecuadas a las necesidades reales de sus territorios (Centro de Educación para el Desarrollo, 2015).

Así, y teniendo en cuenta los cambios de 2014, los temas que ocuparon las agendas de los líderes del proyecto durante 2015 y 2016 estuvieron orientados a las nuevas masculinidades, el análisis multicultural (que incluía la participación de indígenas, gitanos y comunidades afro), la diversidad sexual, la filosofía de la no violencia como propuesta de paz, la perspectiva de género desde el feminismo y el masculinismo, las competencias ciudadanas en las Pruebas Saber Pro, los avances y retrocesos del proceso de paz en Colombia, la democracia participativa en Uniminuto y los derechos y deberes de las comunidades LGTBI (Hernández Parra, 2015). Para ese período, las presentaciones de las conferencias empezaron a intercalarse entre los estudiantes, los docentes del CED y el personal externo a la institución. Con ese cambio, el perfil de las cátedras se orientó hacia la consolidación del proyecto de ciudadanía a partir de la integración de los procesos con actividades de indagación y profundización. Para el año 2016, se creó un semillero de investigación sobre multiculturalidad para que integrara las temáticas abordadas en las cátedras. En ese sentido, el proyecto de ciudadanía contenía actividades en la semana de la ciudadanía (celebradas entre el 14 y 19 de marzo), tareas en la semana de competencias ciudadanas (realizadas entre el 11 y 13 de abril), eventos en la semana de la sociedad civil colombiana (efectuadas entre el 10 y 13 de mayo), socialización de las experiencias en el semillero y una salida pedagógica que sirviera de socialización en campo del trabajo realizado en la vereda San Andrés de Girardota, la cual se estaba adelantando desde el semillero (Hernández Parra y Lenis Sucerquia, 2016).

El perfil temático de las cátedras del primer semestre de 2017 estuvo orientado a la evolución de la ciudadanía y sus implicaciones, la organización y la participación ciudadana, las veedurías ciudadanas y la afrocolombianidad. Para ese momento, la líder del proyecto de ciudadanía era la docente Janet de la Cruz Agudelo Carmona. Sus esfuerzos buscaron reconocer la realidad a partir de variables interpretativas como los cambios históricos que han perfilado el ejercicio de la ciudadanía, la importancia de la democracia participativa en los asuntos públicos (mediante la búsqueda del cumplimiento de los programas de gobierno), los mecanismos democráticos de representación que facilitan la vigilancia de la gestión pública y el reconocimiento del valor cultural y étnico de las comunidades negras colombianas con huella africana. La insistencia por impulsar la reflexión sobre esas temáticas buscaba que los estudiantes conocieran y cuestionaran la realidad de la que hacían parte. De esta manera, se generaron emociones que activaran el análisis crítico sobre esas problemáticas desde un posicionamiento objetivo que excitara la transformación social. En ese sentido, las cátedras se fueron convirtiendo en un espectro de discusión que abrió la 
puerta a una ética cívica, liderada desde la institución, para complementar los adelantos educativos realizados en las aulas de clase. Así, se promovió la consolidación de sociedades incluyentes y equitativas, y se buscó articular el pluralismo moral de las sociedades actuales.

\section{Conformación del ciclo de formación ciudadana}

En realidad, la estrategia del proyecto de ciudadanía lograba integrar actividades y eventos que apostaban a la formación ciudadana. Sin embargo, esa estrategia adolecía de un documento proyectivo que orientara los procesos. No obstante, ese problema fue resuelto en el segundo semestre de 2017, cuando el equipo administrativo (conformado por el director Bedoya Bayer, la secretaria académica Ilba Patricia Rodríguez Escobar, la coordinadora de investigación Natalia Guacaneme Duque y la coordinadora administrativa Rodas Restrepo) realizó unas jornadas de planeación que dieron como resultado la conformación de macroprocesos para las actividades de proyección y extensión. Estos macroprocesos reestructuraron los macroproyectos de 2014 y facilitaron el diseño de un modelo de gestión basado en procesos que reorganizó las tareas realizadas por el CED desde el afianzamiento de sus componentes, a saber: consolidación del CED, excelencia académica, excelencia investigativa, proyección social y extensión. Con ello, se buscó una consistencia estratégica que reclasificara las funciones operativas a la luz del Plan de Desarrollo, 2013-2019 (Corporación Universitaria Minuto de Dios, 2014) y en clave de la constitución de planes formativos de alta calidad. Así, los servicios ofrecidos por el CED sentaron las bases de su trabajo y le apostaron al alcance de una educación superior de alta calidad con un elevado desempeño en el componente específico de competencias ciudadanas y un prominente nivel de cualificación docente. Estos tres elementos quedaron expresados en macroactividades que orientaron la nueva visión prospectiva del CED.

Para el caso de competencias ciudadanas, las macroactividades fueron expresadas en: i) olimpiadas ciudadanas, ii) aula virtual de competencias ciudadanas, iii) ciclo de formación ciudadana y iv) jornada por la paz. Con esa estructura, el ciclo de formación ciudadana pasó a reemplazar el proyecto de ciudadanía para ser conformado por eventos como las cátedras, los debates, los cine-foros, lo talleres experienciales y los eventos de liderazgo en valores en un solo paquete. Con ese diseño estratégico, la apuesta debió estar estructurada en un proyecto que fuera la carta de navegación de las actividades. Por eso, y para ese mismo año, el docente Andrés Felipe Castañeda Jiménez organizó el proyecto con una estructura que incluyó la justificación, el objetivo general, los objetivos específicos, el marco teórico y la bibliografía. Con ese material, la articulación de las tareas del ciclo facilitaría el seguimiento, la construcción de 
indicadores y el alcance de los impactos. Empero, y más allá de esa proyección administrativa, el ciclo se aprestaba como un espacio para

[p]ensar el mundo, sus dolores, sus desavenencias, el surgimiento de constantes fenómenos que movilizan el trasegar humano: transformándolo, confrontándolo, situándolo en el plano de una realidad a veces controversial, es un escenario obligado y puesto para ser visto, observado, escuchado, sentido, vivenciado, atravesado por las confrontaciones de la palabra y dignificado en acciones de ciudadanos comprometidos, insatisfechos con la monotonía de una realidad a veces poco favorecedora de acciones humanas emancipatorias y alejadas, en grandes ocasiones, de una visión ética y estética de mundo que promueva compromisos hacia el respeto de los derechos humanos, la ciudadanía, la no violencia, la realización de la justicia y la vivencia de la libertad y la solidad responsable. (Castañeda Jiménez, 2017, p. 2)

En otras palabras, el ciclo de formación ciudadana dispuso las condiciones necesarias para generar un espacio de pensamiento entre los estudiantes, la planta docente y el cuerpo administrativo de la Uniminuto, sede Bello. En este contexto, se reflexionó sobre los fenómenos que hacían parte de los macro y microcontextos de la sociedad colombiana para promover ambientes académicos que centraran la atención en la discusión sobre las nuevas realidades, la movilización de los discursos y la procura de una ciudadanía activa. Estos espacios en formato de ágora abrieron el debate sobre las múltiples realidades con el objetivo de generar alternativas movilizadoras de mundo y fomentar posiciones diversas. Bajo esas pretensiones, el lugar de los conferencistas dejó de ser un escenario propio de los estudiantes y pasó a ser un espacio para los expertos en diferentes ramas, pues el objetivo se reorientó al afianzamiento, actualización y novedad de los conocimientos de esos especialistas que se encontraban en ejercicio y que podían contribuir al aprendizaje de los estudiantes desde sus experiencias. Con ello, el ciclo buscó facilitar el conocimiento de diferentes fenómenos sociales, incentivar el debate discursivo y fomentar intereses que suscitaran la construcción de nuevas realidades.

Con el formato de las macroactividades, el cumplimiento de la visión prospectiva de la formación ciudadana modeló los procesos del CED de Bello. La conformación del ciclo temático de ciudadanía se convirtió en la mejor alternativa para invitar a los estudiantes a aprender a aprender, estimular el pensamiento, suscitar el entendimiento, promover la transferencia de lo conocido, sugerir la resolución de los problemas y estimular la creación de un mundo mejor. Con esa estrategia de aprendizaje, el conocimiento cívico siguió un proceso espiral representado en la continua búsqueda de información y en el encuentro de la funcionalidad de los significados de lo que se estaba aprendiendo. Bajo esa perspectiva, el ciclo se aprestó como la mejor herramienta para contribuir a la formación humana de los estudiantes y abonar su preparación para la vida, la ciencia y la cultura. Se combinaron, entonces, los principios que rigen al CED 
de Bello con los aportes realizados por Bess Altwerger y Bárbara M. Flores (1994). Recordemos que el término ciclo temático fue utilizado por ellas dos en el artículo "Theme cycles: creating communities of learnes" para reorientar la enseñanza de los estudiantes desde la integración curricular con espacios de participación democrática que estimularan el aprendizaje colaborativo. En ese sentido, la reorganización del proyecto de ciudadanía por ciclos se convirtió en la mejor alternativa para lograr un acercamiento integral de los estudiantes a los temas de urbanidad y civismo. Las actividades que hicieron parte de ese formato se fueron consolidando en espacios de apertura enfocados a la formación cívica para promover puentes de conexión que han entrelazado las problemáticas que han hecho parte del ser ciudadano y la ciudadanía.

Desde esa estructura, las cátedras pasaron a ser una especie de poliatría dedicada a la reflexión de los problemas colombianos; a la mejor manera de la Cátedra de Formación Ciudadana Héctor Abad Gómez de la Universidad de Antioquia (Universidad de Antioquia, 2010, p. 100). Cada sesión incentivó el rechazo a la intransigencia excluyente y estimuló el lenguaje ético-civil como medio para resolver los retos de la sociedad contemporánea. La disposición de ese espacio académico se ha ido convirtiendo en una estrategia efectiva del CED que ha logrado potenciar el proceso de enseñanza-aprendizaje iniciado en las aulas de clase. Se combinó, así, la función característica de enseñanza de los cursos ofrecidos por la sede con el refuerzo de escenarios que aportan a la construcción de civilidad mediante la reflexión pública sobre la realidad colombiana. En tal sentido, la fundamentación de las cátedras abiertas de ciudadanía se aprestó como el momento más propicio para corroborar el valor de la palabra en ciernes a la circulación de ideas que garantizaran la discusión pluralista y democrática de las posiciones. De esta manera, se buscó superar la fragmentación de los temas de ciudadanía y se aprovecharon los referentes de diálogo (expertos en las temáticas) para facilitar la interacción colectiva en torno al civismo y los espacios de convivencia social entre las personas, incluyendo temáticas como la educación ambiental.

\section{Ciclo de formación ciudadana y jornada por la paz: unidos por un mismo resultado}

Con el terreno abonado por los cambios administrativos, el ciclo de formación ciudadana se vio potenciado por las actividades realizadas durante 2018. Para ese año, los temas que fueron objeto de discusión en el formato de las cátedras estuvieron relacionados con el compromiso social ciudadano, la evolución conceptual de la ciudadanía, la posición ética del amor, el conocimiento de los planes de gobierno de los aspirantes a la presidencia de la república, la resolución de conflictos desde el código de policía, los mecanismos de participación ciudadana en el marco del neoconstitucionalismo, las experiencias de 
ecoturismo accesible en Costa Rica (como experiencia modelo para replicar el turismo incluyente en personas con discapacidades diversas en Colombia), la participación ciudadana juvenil y la cultura política en los jóvenes. Estos temas buscaron promover el fortalecimiento de la población estudiantil en las competencias ciudadanas a partir de la reflexión abierta sobre las acciones políticas, sociales, económicas y culturales que han hecho parte de la idiosincrasia colombiana, lo que facilitó la generación de ambientes de discusión y aprendizaje y suscitó el análisis de la estructura social bajo un principio de priorización que hizo énfasis en los elementos constitutivos de construcción de comunidad. Desde esa orientación, las discusiones sobre el papel que debería cumplir un buen ciudadano en la localidad a la que pertenece, el respeto de los derechos propios y del otro, el reconocimiento en la participación cívica y el compromiso del ciudadano en todos los entornos modeló cada una de las reflexiones que hicieron parte de las cátedras.

Esos esfuerzos fueron complementados con las actividades hechas en el marco de la jornada por la paz (celebrada entre el 19 y 30 de septiembre), ya que los debates (en formato conversatorio), cine foros, talleres experienciales y eventos de liderazgo en valores se hicieron en llave con la jornada de manera colaborativa. Los dos docentes-líderes de las macroactividades (Elidio Alexander Londoño Uriza y Luis Carlos Carvajal Vallejo respectivamente) unieron esfuerzos para incentivar el conocimiento de los factores que influyen en la participación ciudadana de los jóvenes, promovieron la reflexión del proceso de posacuerdo colombiano (facilitando la participación de las voces de las víctimas del conflicto armado, en particular las de Inis Johana Mosquera Reyes y Yolanda Perea) $)^{5}$ e insistieron en la deliberación del tema de la paz en la dinámica de la no violencia y bajo la lógica de la superación de los conflictos. Con la unión de las dos macroactividades (ciclo de formación ciudadana y jornada por la paz), las apuestas por la conformación de un ethos de formación integral se vieron allanadas. El trabajo colaborativo potenció la planeación táctica del CED en materia de competencias ciudadanas y reforzó los propósitos de propiciar aptitudes de autonomía, buena ciudadanía, excelencia profesional y opciones éticas cívicas de sana convivencia. Cabe agregar que, para ese evento, Bienestar Institucional también hizo parte del trabajo colaborativo.

La unión de las macroactividades no fue un asunto de azar o de circunstancia coyuntural. Desde finales de 2017, la administración del CED de Bello diseñó una ficha estratégica de planeación táctica participativa para consolidar los procesos (Centro de Educación para el Desarrollo, 2017). Dentro de esa propuesta, la

Esto se hizo en el marco de los acuerdos de paz que el Gobierno del expresidente Juan Manuel Santos Calderón llevó a cabo con la guerrilla de las Fuerzas Armadas Revolucionarias de Colombia - Ejército del Pueblo (FARCEP) en noviembre 24 de 2016, para ponerle fin al conflicto armado colombiano iniciado en 1960. 
visión prospectiva incentivó la articulación de las tres funciones sustantivas del CED: docencia, investigación y proyección social. En ese sentido, el plan táctico construido para 2018 y 2019 se adaptó al tablero de mando integral diseñado por David Norton y Robert Kaplan en 1990 para potenciar los activos intangibles del CED (que no se habían podido medir en la visión y estrategia diseñadas hasta ese momento) ${ }^{6}$. Con ese modelo de gestión, las prácticas gerenciales de la unidad académica buscaron consolidar las apuestas de trabajo en las líneas de responsabilidad social y ciudadanía activa, lo que favoreció la realización y ejecución de planes que contribuyeran a la configuración del CED como un centro de excelencia. Así, el camino estaría abonado para consolidar al Centro como un agente de desarrollo que promoviera el protagonismo de las personas, organizaciones y comunidades en los procesos de transformación social y en los mecanismos de construcción de paz en los territorios. Por eso, la unión de las macroactividades supuso el afianzamiento de las actividades para ampliar el rango de acción y efectividad en los procesos.

\section{Perspectivas y orientaciones de trabajo del ciclo de ciudadanía}

La experiencia con la Jornada por la paz abrió las puertas para que la proyección del plan táctico fuera efectiva en la ejecución del ciclo de formación ciudadana. El trabajo hecho entre el CED y Bienestar Institucional durante el segundo semestre de 2018 sirvió de antecedente para vincular a las demás dependencias a las actividades del ciclo. Por eso, las experiencias del primer semestre de 2019 se realizaron bajo la alianza entre la Facultad de Ciencias Humanas y Sociales y la Facultad de Ciencias Económicas y Administrativas. Los temas que fueron objeto de discusión estuvieron centrados en los elementos históricos de la histeria, los límites planetarios y el Antropoceno, así como la convivencia y seguridad ciudadana en el área metropolitana del Valle de Aburrá. Así, los esfuerzos aunados buscaron apoyar, consolidar y fortalecer los procesos adelantados por el CED en materia de educación cívica (en particular desde la estrategia del ciclo), ya que la ciudadanía es un tema de interés general que involucra a todas las áreas y facultades de la institución. Desde luego, el trabajo combinado se convirtió en la mejor oportunidad para establecer una cultura ciudadana sólida que priorizara en la instauración continua de consensos que hiciera especial énfasis en la implementación de buenas conductas, la acentuación de los valores sociales, el buen manejo de actitudes comportamentales y el respeto a las diferentes percepciones de los miembros de la sociedad colombiana.

Ese perfil de colaboración y unión de esfuerzos fue replicado en el segundo semestre de 2019. No obstante, la orientación temática de las cátedras abier-

Hay que advertir que la ficha fue adaptada del Ministerio de Educación Nacional, en particular de la guía 28 que hace énfasis en la gestión de buenas prácticas (Ministerio de Educación Nacional, 2007, p. 20). 
tas de ciudadanía estuvo centrada en el bicentenario de la Batalla de Boyacá (1819-2019) a través de enfoques como la iconografía y comunicaciones en la Independencia, además de una muestra musical y gastronómica de las regiones de Colombia. En realidad, todo el sistema Uniminuto, a nivel nacional, fue integrada al programa Inspirando país. Colombia 200 años para incentivar la apropiación de la historia común de los colombianos. Así, las diferentes áreas, dependencias, facultades y departamentos de la institución se unieron a los actos conmemorativos generados por el bicentenario del nacimiento del país como una nueva nación. Bajo ese horizonte, los ejes temáticos que modelaron los eventos fueron: memorias de 1819, los rostros de doscientos años de historia, doscientos años de retos y avances, e inspirando país. En otras palabras, las actividades de toda la institución universitaria se condensaron en hacer un ejercicio holístico de recuperación de la memoria histórica nacional, lo cual hizo que la integración de las estrategias fuera mucho más sólida.

Para el año 2020, las líneas de reflexión del ciclo (fundamentadas desde las cátedras del CED) incluyeron temáticas de interés general como la intervención social en el marco de la interculturalidad colombiana y la seguridad alimentaria en el contexto de la pandemia por la COVID-19. En efecto, el ciclo buscó estimular la reflexión actualizada de las problemáticas que hacen parte del país, y del mundo en general. Con ello, se buscó contribuir a la generación de criterios de pertinencia y eficacia que potencien el mejoramiento del desarrollo humano integral de la sociedad. Bajo ese marco, el contexto de la pandemia por la COVID-19 influyó en la disgregación de esas problemáticas y en la necesidad urgente de discutir las exigencias contextuales que deparó la enfermedad.

Hay que advertir que el plan táctico del CED (diseñado para 2018 y 2019) se ha ido nutriendo de la cadena de valor (reestructurada en 2019) de la institución, la cual ha propendido por modernizar e integrar el sistema Uniminuto para generar una cultura que incluya buenas prácticas dentro de la gestión de los procesos y promover la implementación de un sistema integrado entre calidad académica y optimización eficiente de los procedimientos. Bajo ese decorado institucional, la cultura ciudadana promovida por el CED de Bello (basada en el formato de ciclo temático) se ha inclinado hacia la formación de una calidad de vida fundamentada en la sana convivencia cívica que facilite la distinción de los derechos y deberes que tienen los colombianos y estimule la reflexión crítica y activa frente al reconocimiento de la realidad. De esta maneras, se busca consolidar una conciencia fonémica de lo que significa ser ciudadano con conciencia y responsabilidad social.

En ese orden de ideas, la propuesta de un ciclo basado en la ciudadanía reorganiza los subtemas que hacen parte de la formación ciudadana y posibilita 
el diálogo con los sistemas y estructuras locales, nacionales e internacionales. Además, pone en comunicación los problemas que afectan la interacción y conectividad de las comunidades, e incentiva el debate sobre las dinámicas del poder, los niveles de la identidad poblacional, la importancia de la diferencia, el respeto a la diversidad, las medidas (jurídicas e institucionales del poder público) que inciden en las acciones individuales y colectivas para el trabajo comunitario, las implicaciones del comportamiento éticamente responsable y la importancia del compromiso cívico y activo desde la actuación. Así, el ciclo ha buscado (y sigue buscando) superar los postigos de una ciudadanía centrada en las elecciones, el sufragio y la representación política para abrirle paso a una conciencia ciudadana global, multicultural, activa y social bajo albricias de una humanidad más solidaria y sensible ante sus otros y el mundo que lo rodea.

\section{Conclusiones}

Las estrategias implementadas por el CED de Bello, en materia de formación ciudadana, nos han llevado a una multiplicación de apuestas que han facilitado la apropiación del enfoque de la EPD y el fortalecimiento de la gestión estratégica de sus unidades. Si bien los CED propenden por el aprendizaje integral de la población institucional (y fuera de ella), al interior de sus planes tácticos de trabajo también hay una variopinta forma de funcionamiento que democratiza los campos de acción de cada una de las unidades. En ese sentido, la valoración de las apuestas sugiere la revisión de la labor adelantada en relación con la promoción de los valores cívicos, el reconocimiento del país, el acercamiento a la legislación que nos rige, las formas de participación ciudadana y los medios que posibilitan impactar a las comunidades. Todos estos temas se enmarcan en el diseño y la construcción de tradiciones que fomentan una cultura para la convivencia. El caso del ciclo de formación ciudadana del CED de Bello, por ser un espacio que involucra el aprendizaje continuo y no formal (al no depender de ningún curso o programa académico), permite visualizar el alcance y las limitaciones que el enfoque de la formación ciudadana puede tener en la práctica.

La transversalidad del modelo de aprendizaje cívico en la Uniminuto es pieza garante para que las puestas en escena varíen en su intencionalidad de promover la conformación de una sociedad igualitaria y solidaria, facilite la concientización de la reducción de las brechas sociales y estimule el ejercicio de la ciudadanía sin distingos de condición o situación social. El ciclo de formación ciudadana en Bello es un aporte a los esfuerzos del CED por consolidar una enseñanza integral en los estudiantes. No obstante, la poca dialéctica interregional entre las apuestas de trabajo de cada uno de los centros supone realizar estudios comparados que permitan evidenciar las potencialidades y limitaciones de cada una de las estrategias llevadas a cabo. Además, las exi- 
guas alianzas interinstitucionales con otras universidades no permiten hacer un comparativo sobre la efectividad de las apuestas, pues es de recordar que los procesos de enseñanza de la Uniminuto son sui géneris en sus estructuras y metodologías académicas (respecto a las demás instituciones de educación superior del país), ya que centran su atención en la labor social, la inclusión de la población vulnerable y le apuesta a la apertura de oportunidades educativas. Por ello, y para abrir camino a ese tipo de investigaciones, la reflexión que realizamos se apresta como un ejercicio de revaloración para otear el proceso formativo ciudadano en todos los CED de la Uniminuto.

Sin duda, la breve comprensión que hemos logrado suscita la realización de futuras investigaciones sobre la formación ciudadana en Uniminuto, pues el diálogo académico e investigativo entre las estrategias de cada una de sus seccionales, sedes, facultades y centros académicos facilita el ejercicio de reconstrucción de la memoria histórica y la búsqueda de una justicia social. Así, promueve la visibilización de los problemas que afectan a las minorías étnicas, a las mujeres, a los niños y niñas, a los líderes comunitarios, a las personas en condición de discapacidad y, en general, a la población en situación de vulnerabilidad social. Esto quiere decir que se apunta a los temas que hacen parte de una ciudadanía activa. Es de recordar que nuestro estudio apenas logra abarcar el período 2006-2020, ya que el CED de Bello apenas tiene catorce años de funcionamiento, pero sus campos de acción se han posicionado rápidamente dentro y fuera de la institución. En ese sentido, y teniendo en cuenta la efectividad de su trabajo, la valoración y revaloración de sus apuestas permiten reconocer los aportes que el CED de Bello hace a la formación ciudadana dentro del sistema nacional de la Uniminuto. Este distingue de las demás unidades en relación con sus puestas en escena, que igual se orientan bajo el enfoque de la EPD y la educación popular, pero tien el sello diferenciador de sus estrategias.

En el barrido que hemos realizado es claro que la transversalidad de las estrategias de trabajo del CED de Bello permite la alineación táctica de las macroactividades que hacen parte de la unidad. La labor mancomunada llevada a cabo entre los docentes que lideraban el ciclo de formación ciudadana y la jornada por la paz durante el año 2018 es una clara muestra de ello. Por eso, suponer la articulación entre los procesos de investigación del CED con los espacios que brinda el ciclo sugiere la conformación de escenarios que permitan la divulgación de los resultados de indagación. Además, los adelantos de los proyectos de intervención social también acucian la disposición de espacios que posibiliten la difusión del trabajo que se desarrolla en los proyectos, y en esa dirección el ciclo igualmente es una ventana para divulgar esa labor a toda la comunidad universitaria. Esta ventana también puede servir para promocionar el trabajo del voluntariado que el CED lidera con organizaciones dedicadas a las personas en riesgo de exclusión 
social. Esas alianzas inter-macro-actividades podrían dar pie a uniones interinstitucionales con otras universidades que abonen camino para eventos, como el primer congreso nacional de ciudadanía en el marco de la formación ciudadana. Pero eso solo es posible si los esfuerzos prospectivos tienen asidero en la realidad. Por lo pronto, el ciclo es el paso inicial para todas esas apuestas.

Aunque en el presente escrito no ampliamos la mirada de observación a las temáticas que han hecho parte del ciclo, las alusiones que hicimos permiten sugerir la incorporación de elementos de análisis que también hacen parte de la formación ciudadana. En algunos casos, esos elementos pueden ser líneas de profundización que faciliten el entendimiento de los organismos de representación de los colombianos. tales como el funcionamiento de la Cámara de Representantes, las actuaciones de los concejales, los conciertos de los senadores, la forma para acceder a esos espacios de representación pública y demás aspectos del ámbito político colombiano. En otros casos, la introducción de asuntos de interés general igualmente hace parte del aprendizaje cívico de la comunidad universitaria.: la situación geopolítica mundial para comprender los procesos sociales, políticos, económicos y culturales que ocurren en el país y que no son lejanos a las problemáticas del globo terráqueo. En realidad, ese tipo de orientaciones temáticas son un buen complemento para ampliar los niveles de observación de aspectos como la pobreza, la exclusión, la desigualdad social, la inequidad de género, la violencia y el medio ambiente, pues permiten reforzar los esfuerzos de formación integral liderados por el CED.

En efecto, el ciclo de formación ciudadana es una apuesta en constante construcción que promueve el posicionamiento del aprendizaje cívico de la población estudiantil del CED de Bello. Los adelantos que hasta el momento se han hecho cimientan las bases de la estrategia y son el punto de partida para mejorar sus condiciones de aplicación táctica. Desde ese escenario, el reconocimiento de los valores ciudadanos, el aprendizaje de las condiciones del país, la estructura de la legislación que nos rige y los mecanismos de participación son algunos de los temas que promueven la participación proactiva y facilitan el impacto a las comunidades de proveniencia de la comunidad estudiantil. Estos temas sugieren la anuencia de los estudiantes para multiplicar las experiencias y orientar los aprendizajes hacia la transformación territorial y social, así como aprovechar sus entornos familiares, de pareja, laborales, comunales y barriales. Así, el ciclo es un escenario que propicia y aumenta la interacción entre los estudiantes, profesores, administrativos y público en general para universalizar el aprendizaje desde la discusión crítica, reflexiva, propositiva, interdisciplinaria, autónoma y responsable sobre lo que compete ser ciudadano socialmente responsable y éticamente comprometido con la 
sociedad. Por supuesto, todo esto se realiza bajo un modelo estratégico de planeación táctica participativa.

Si bien en el escrito no profundizamos en la planeación táctica diseñada por la administración del CED de Bello en 2018 (que creó los macroprocesos de acción, entre ellos el ciclo de formación ciudadana), la alusión que hicimos supuso la contextualización de las estrategias de articulación hasta el año 2020, pues la visión prospectiva de esa carta de navegación consideró su actualización luego de la socialización del Plan de Desarrollo del Sistema Uniminuto para 2020-2025. El hecho de no ahondar en esa herramienta administrativa no nos impide reconocer su funcionalidad para potenciar las capacidades de las apuestas de trabajo de la unidad. El estudio sobre el ciclo (que es una de las macroactividades de mayor perfil de actuación del CED de Bello) también es una oportunidad para revisar y reinterpretar esos lineamientos, ya que puede ser insumo para considerar los logros alcanzados y los retos que hacen parte del campo de acción del CED y de la formación ciudadana en particular. Esta afirmación se basa en las evidencias que deja la experiencia del ciclo para reforzar el trabajo adelantado e incluir elementos de acción que consoliden la estrategia. Así, se sugieren horizontes de diálogo interinstitucional para ampliar las perspectivas futuras de desarrollo y fortalecer los retos que la proyección social de la unidad demanda.

En síntesis, haber intentado considerar el ciclo de formación ciudadana del CED de la Uniminuto, sede Bello, sirve para visualizar una experiencia estratégica que aporte a las apuestas formativas de la institución. Acudir a este tipo de reflexiones permite reconsiderar las hipótesis que deja el modelo de educación de la Uniminuto y actualizar las prácticas administrativas, pedagógicas y de proyección social implementadas en las sedes. Así pues, el artículo también es un ejercicio que vale para reconocer el pasado histórico del CED y la manera en que alinea sus campos de acción a la estrategia institucional. Todos esos elementos coadyuvan a la renovación permanente y dan cabida a posibles serendipias de innovación investigativa que potencien el ejercicio formativo de la unidad. En tal sentido, el análisis sobre los avances del ciclo y su contribución académica adquirirá, en el tiempo, una condición de referencia para la visión prospectiva del CED. Además, proporciona aspectos significativos para los procesos de aprendizaje cívico de otras instituciones de educación superior, las cuales igualmente podrán aprovechar la reflexión que realizamos bajo una relación de interacción entre la propuesta del CED y la aplicación de la formación ciudadana que estén liderando, pues la apertura y el diálogo de las experiencias amplían los niveles de interpretación de la enseñanza en diferentes escenarios de aprendizaje. Estos escenarios finalmente ayudarán a consolidar el perfil de la educación cívica en el país. 


\section{Agradecimientos}

El artículo es resultado del proceso de gestión adelantado para las cátedras abiertas de ciudadanía que el Centro de Educación para el Desarrollo (CED) de la Corporación Universitaria Minuto de Dios (Uniminuto), sede Bello, ha llevado a cabo desde el área de Proyección Social y Extensión, y bajo el marco del Ciclo de Formación Ciudadana entre 2017 y 2020.

\section{Referencias}

Altwerger, B. y Flores, B. (1994). Theme Cycles: Creating Communities of Learners. Primary Voice K-6, Challenge for Challenge: Theme cycles, 2(1), 2-6.

Botero Carrillo, P, Morán Matiz, A. y Solano Salinas, R. (2010). Fundamentos conceptuales del CED. Educación para el Desarrollo, Formación Ciudadana y Responsabilidad Social Universitaria. Corporación Universitaria Minuto de Dios.

Castañeda Jiménez, A. (2017). Cátedras de ciudadanía. CED -Centro de Educación para el Desarrollo. Seccional Bello [manuscrito no publicado]. Centro de Educación para el Desarrollo, Corporación Universitaria Minuto de Dios.

Chaux Torres, E., Lleras Acosta, J y Velásquez, A. (comps.). (2004). Competencias ciudadanas: de los estándares al aula. Ministerio de Educación Nacional -Departamento de Psicología y CESO, Universidad de los Andes.

Centro de Educación para el Desarrollo. (2017). Ficha para sistematización de una buena práctica [manuscrito no publicado]. Corporación Universitaria Minuto de Dios Sede Bello y Centro de Educación para el Desarrollo (CED).

Centro de Educación para el Desarrollo. (2015). Diplomado en ciudadanía y liderazgo social. Corporación Universitaria Minuto de Dios.

Centro de Educación para el Desarrollo. (2014). Plan de acción -Plan operativo 2014. Corporación Universitaria Minuto de Dios.

Corporación Universitaria Minuto de Dios, Uniminuto. (2015). Memorias estadísticas Uniminuto 2015. Dirección de Planeación y Desarrollo; Uniminuto.

Corporación Universitaria Minuto de Dios, Uniminuto. (2014, 9 de septiembre). Acuerdo No. 02. Por el cual se aprueba y promulgan los lineamientos generales para la proyección social. Corporación Universitaria Minuto de Dios.

Corporación Universitaria Minuto de Dios, Uniminuto. (2014). Uniminuto. Plan de Desarrollo, 2013-2019. Corporación Universitaria Minuto de Dios.

Corporación Universitaria Minuto de Dios, Uniminuto. (2006, 6 de marzo). Acuerdo 224. Por el cual se autoriza el cambio de denominación del Centro de Práctica Social como Centro de Educación para el Desarrollo, y se reestructura su organización interna y sus funciones. Corporación Universitaria Minuto de Dios.

Corporación Universitaria Minuto de Dios, Uniminuto. (s. f.). Antioquia y Chocó. ¿Qué hacemos? Corporación Universitaria Minuto de Dios. http://www.uniminuto.edu/web/seccionalbello/nosotros11 
Cortina-Orts, A. (2010). Justicia cordial. Trotta.

Estudiantes del Diplomado (2015). Diagnóstico participativo. Diplomado en Ciudadanía y Liderazgo Social [manuscrito no publicado]. Corporación Universitaria Minuto de Dios Sede Bello, Centro de Educación para el Desarrollo.

Freire, P. (1972). La pedagogía del oprimido. Siglo XXI.

Hernández Parra, E. D. y Lenis Sucerquia, L. (2016). Programación del proyecto de ciudadanía para 2016-I [manuscrito no publicado]. Corporación Universitaria Minuto de Dios Sede Bello, Centro de Educación para el Desarrollo.

Hernández Parra, E. (2015). Cátedras Abiertas de Ciudadanía 2015-2 [manuscrito no publicado]. Corporación Universitaria Minuto de Dios Sede Bello, Centro de Educación para el Desarrollo.

McLaughlin, T. H. (1992). Citizenship, diversity and education: a philosophical perspective. Journal of Moral Education, 21(3), 235-250. https://doi.org/10.1080/0305724920210307

Mesa Peinado, M. (dir.). (2000). La educación para el desarrollo en la comunidad de Madrid: tendencias y estrategias para el siglo XXI. Informe a la Dirección General de Cooperación y Voluntariado de la comunidad de Madrid. Centro de Investigación para la Paz y Mimeo.

Ministerio de Educación Nacional. (2007). Aprendizajes para mejorar. Guía para la gestión de buenas prácticas, guía 28. Ministerio de Educación Nacional de la República de Colombia.

Morán Matiz, A. (2010). Un modelo de formación ciudadana -Soporte de procesos de transformación social. Revista Prospectiva, 15, 105-131. https://doi.org/10.25100/prts.v0i15.1107

Morán Matiz, A., Donato Hernández, A. e Izquierdo Ramírez, P. (2009). Caja de herramientas para la formación ciudadana, serie educación para el desarrollo. Corporación Universitaria Minuto de Dios, Centro de Educación para el Desarrollo.

Moya Garzón, Y. (2012). ¿Cómo va la formación ciudadana? Seguimiento a la implementación del Modelo de Formación Ciudadana del CED de Uniminuto. Polisemia, 8 (14), 124-125. https://doi. org/10.26620/uniminuto.polisemia.8.14.2012.124-125

Organización El Minuto de Dios. (2013). P. Rafael García Herreros. (Cúcuta, enero 17 de 1909 - Bogotá, noviembre 24 de 1992). Organización El Minuto de Dios, Provincia Eudista Minuto de Dios. https:// www.minutodedios.org/documentos/padre_rafael_garcia_herreros_biografia.pdf

Orrego Echeverría, I. (2014). Del desarrollo social y humano a la noción del "Buen Vivir"; trasegar reflexivo sobre el desarrollo en las construcciones conceptuales del CED-Uniminuto. En E. de Villa (coord.). Responsabilidad social de las universidades, tomo $V$ (pp. 165-191). Red Latinoamericana de Cooperación Universitaria.

Ramos Pino, J., Ortiz Palacio, P. y Gómez Cardona, Y. (2013). Uni juventud [manuscrito no publicado]. Corporación Universitaria Minuto de Dios. https://es.calameo.com/read/0028424937975c6712859

Riquelme-Muñoz, S. (2018). Citizenship Curriculum Development in Chile and Argentina during the 1990s and the 2000s: Patterns and Justifications [Doctoral thesis, University of Melbourne, Australia]. Minerva Access. https://minerva-access.unimelb.edu.au/handle/11343/219374

Solano Salinas, R. (2011). Qué es Educación para el Desarrollo para el CED-UNIMINUTO [Manuscrito no publicado]. Corporación Universitaria Minuto de Dios. https://www.academia.edu/31790974/ Qu\%C3\%A9_es_Educaci\%C3\%B3n_para_el_Desarrollo_para_el_CED_UNIMINUTO 
Solano Salinas, R. (2016). Educación para el desarrollo: una mirada desde el sur por la construcción de una educación para el cambio. Ánfora, 18 (30), 87-120. https://doi.org/10.30854/anf.v18.n30.2011.92

Universidad de Antioquia. (2010). Cátedra de Formación Ciudadana Héctor Abad Gómez. Un aporte a la construcción de civilidad. Memorias 2008-2009. Universidad de Antioquia, Corporación Héctor Abad Gómez y Apotema S. A. S.

Valencia Rivas, J. (2013). Entre la realidad y el desafío de una práctica en responsabilidad social. Tendencias \& Retos, 19(1), 71-86. https://dialnet.unirioja.es/servlet/articulo?codigo $=4929337$

Vicerrectoría General Académica (2018). Lineamientos generales. Estrategias de Proyección Social. Uniminuto. Xpress Estudio Gráfico y Digital S.A.S., Corporación Universitaria Minuto de Dios. 